\title{
Adaptive Fixed-Time Non-singular Terminal Sliding Mode Attitude Stabilization Control for Rigid Spacecraft with Actuator Faults
}

\author{
Zhiguo Han*, Hui Fu, Fanghao Tian* \\ School of Astronautics, Northwestern Polytechnical University, Shaanxi, Xi'an 710072, China. \\ * Corresponding author. Tel.: +8618066611835, +8618829238627; email: zghan2017@nwpu.edu.cn, \\ tianfanghao1995@163.com \\ Manuscript submitted March 10, 2019; accepted May 21, 2019. \\ doi: 10.17706/ijcee.2019.11.3.155-163
}

\begin{abstract}
Fixed-time convergence control strategies based on adaptive Non-Singular Terminal Sliding Mode are proposed for rigid spacecraft attitude stabilization subject to actuator faults. A novel fixed-time sliding mode surface is used. Then, a fixed-time controller with adaptive law is derived to guarantee that the closed-loop system is stable in the sense of the fixed-time concept. Finally, the Lyapunov stability analysis shows that the controller has a good fault-tolerant performance on actuator faults. Numerical simulation verified the good performance of the controller in the attitude stabilization control.
\end{abstract}

Key words: Adaptive non-singular terminal sliding mode, attitude stabilization, fixed-time control, rigid spacecraft.

\section{Introduction}

As space technology improves, space flight missions have higher control requirements [1]-[5]. Ref. [6] proposed a novel sliding-mode control laws to stabilize a class of uncertain on-linear systems. Because the sign function often used in the sliding-mode control which can cause chattering of control input, in order to reduce the influence of the sign function, the saturation function is used to take its place in proposed controllers. Ref. [7] investigates the distributed finite-time consensus problem of second-order multi-agent systems (MAS) in the presence of bounded disturbances. Ref. [8] proposed an adaptive non-homogeneous higher order sliding mode control (HOSMC) method for a class of uncertain nonlinear systems., the proposed HOSMC algorithm provide fast convergence rate by using non-homogeneous finite time stabilization when exist a large initial tracking error. In [9], the authors aim at the longitudinal model of an air-breathing vehicle under system uncertainties and actuator failures.

In term of spacecraft attitude control, the finite-time sliding mode using the terminal sliding mode has been proven the efficacy to address the attitude control related issues. In [10], the authors proposed a passive fault-tolerant control by using the finite-time sliding mode control. To tolerate the possible faults of actuator and also to improve the fault tolerant capability, the author [11] proposed an adaptive sliding mode control methodology in the framework of global sliding mode control. With application of this scheme, severe actuator faults can be tolerated, and the attitude tracking error is governed to be asymptotically stable. The attitude tracking maneuver is accomplished in finite-time, the objective of fast slewing maneuvers is thus realized. 
Inspired by [12], on the basis of [13], this paper introduces the fixed-time non-singular terminal sliding mode (FNTSM) control law, it designs a fixed-time fault-tolerant attitude tracking controller based on the parameter adaptive method. The main contributions of this paper are stated as follows:

To achieve FTC for a spacecraft's attitude stabilization, a novel FNTSM control law is designed in the sense of the fixed-time concept, which is robust against external disturbances under actuator faults.

\section{Spacecraft's Attitude Models}

A rigid spacecraft's attitude kinematics and dynamics equations are modeled as follows [14]-[16]:

$$
\left\{\begin{array}{l}
\dot{q}_{0}=-0.5 \boldsymbol{q}^{\mathrm{T}} \boldsymbol{w} \\
\dot{\boldsymbol{q}}=0.5\left(\boldsymbol{q}^{\times}+q_{0} \boldsymbol{I}_{3}\right) \boldsymbol{w} \\
\boldsymbol{J} \dot{\boldsymbol{w}}=-\boldsymbol{w}^{\times} \boldsymbol{J} \boldsymbol{w}+\boldsymbol{D u}(t)+\boldsymbol{d}(t)
\end{array}\right.
$$

where the vector $\boldsymbol{q}=\left[q_{1}, q_{2}, q_{3}\right]^{\mathrm{T}}$ and the scalar $q_{0}$ satisfy the constraint: $q_{0}^{2}+\boldsymbol{q}^{\mathrm{T}} \boldsymbol{q}=1 . \quad \boldsymbol{J} \in \mathbf{R}^{3 \times 3}, \boldsymbol{w} \in \mathbf{R}^{3}$, $\boldsymbol{u} \in \mathbf{R}^{N}, \boldsymbol{d} \in \mathbf{R}^{3} . \boldsymbol{D} \in \mathbf{R}^{3 \times N}$ and $N$ are the parameters of spacecraft. For the specific meaning, please refer to [16]. Define $p^{\times}$as:

$$
\boldsymbol{p}^{\times}=\left[\begin{array}{ccc}
0 & -p_{3} & p_{2} \\
p_{3} & 0 & -p_{1} \\
-p_{2} & p_{1} & 0
\end{array}\right]
$$

Assumption 1: The control input sitisfy $\boldsymbol{u}(t) \leq \boldsymbol{u}_{\max }$, where $\boldsymbol{u}_{\max }$ is the maximum output torque.

According to [12]-[13], we have:

$$
\boldsymbol{u}(t)=\boldsymbol{E}(t) \boldsymbol{u}_{c}(t)+\overline{\boldsymbol{u}}_{c}(t)
$$

where $\boldsymbol{u}_{c}(t)=\left[u_{c 1}, \cdots, u_{c N}\right]^{\mathrm{T}}$ is command control torque; $\boldsymbol{E}(t)=\operatorname{diag}\left(E_{1}(t), \cdots, E_{N}(t)\right)$, which satisfies $0 \leq E_{i}(t) \leq 1 ; \overline{\boldsymbol{u}}_{c}(t)=\left[\bar{u}_{c 1}, \cdots, \bar{u}_{c N}\right]^{\mathrm{T}}$ is drift torque.

With (3), the rigid spacecraft's attitude kinetic equation can be further written as:

$$
\begin{aligned}
\boldsymbol{J} \dot{\boldsymbol{w}} & =-\boldsymbol{w}^{\times} \boldsymbol{J} \boldsymbol{w}+\boldsymbol{D} \boldsymbol{u}(t)+\boldsymbol{d}(t) \\
& =-\boldsymbol{w}^{\times} \boldsymbol{J} \boldsymbol{w}+\boldsymbol{D}\left(\boldsymbol{E}(t) \boldsymbol{u}_{c}(\mathrm{t})+\overline{\boldsymbol{u}}_{c}(\mathrm{t})\right)+\boldsymbol{d}(t) \\
& =-\boldsymbol{w}^{\times} \boldsymbol{J} \boldsymbol{w}+\boldsymbol{D} \boldsymbol{u}_{c}(\mathrm{t})+\boldsymbol{D}\left(\boldsymbol{E}(t)-\boldsymbol{I}_{N \times N}\right) \boldsymbol{u}_{c}(\mathrm{t})+\boldsymbol{D} \overline{\boldsymbol{u}}_{c}(\mathrm{t})+\boldsymbol{d}(t) \\
& =-\boldsymbol{w}^{\times} \boldsymbol{J} \boldsymbol{w}+\boldsymbol{D} \boldsymbol{u}_{c}(\mathrm{t})+\overline{\boldsymbol{d}}(t)
\end{aligned}
$$

where

$$
\overline{\boldsymbol{d}}(t)=\boldsymbol{D}\left(\boldsymbol{E}(t)-\boldsymbol{I}_{N}\right) \boldsymbol{u}_{c}+\boldsymbol{D} \overline{\boldsymbol{u}}_{c}+\boldsymbol{d}(t)
$$

Assumption 2: external unknown disturbances $\overline{\boldsymbol{d}}(t)$ satisfies $\|\overline{\boldsymbol{d}}(t)\| \leq b\left(1+\|\boldsymbol{w}\|+\|\boldsymbol{w}\|^{2}+\|\boldsymbol{w}\|^{2-\eta}+\|\boldsymbol{w}\|^{\eta}\right)$, where $b>0 ; 1<\eta<2$ is the parameter to be designed.

Remark 1: According to Assumption 4.3 in [11], the control law of spacecraft satisfy $\|u(\mathrm{t})\| \leq b_{1}\left(1+\|\boldsymbol{w}\|+\|\boldsymbol{w}\|^{2}+\|\boldsymbol{w}\|^{2-\eta}+\|\boldsymbol{w}\|^{\eta}\right)$, the external disturbances satisfy $\|d(\mathrm{t})\| \leq b_{2}\left(1+\|\boldsymbol{w}\|^{2}\right)$, so, formula (5) satisfies the following equation: $\|\overline{\boldsymbol{d}}(t)\| \leq b\left(1+\|\boldsymbol{w}\|+\|\boldsymbol{w}\|^{2}+\|\boldsymbol{w}\|^{2-\eta}+\|\boldsymbol{w}\|^{\eta}\right)$.

\section{Designing the Fault Tolerant Controller Law}

The control law design is carried out in this section. The control objectives are: 


$$
\lim _{t \rightarrow t_{f}} q_{0}=1, \lim _{t \rightarrow t_{f}} \boldsymbol{q}_{v}=0, \lim _{t \rightarrow t_{f}} \boldsymbol{w}=0
$$

\subsection{Control Design}

Step 1: Designing the sliding mode surface

In this paper, a sliding mode surface is selected as follows [17]:

$$
\boldsymbol{S}=\boldsymbol{w}+\boldsymbol{S}_{a u}
$$

where $S \in \mathbf{R}^{3}$ is the sliding mode surface, $S_{a u}=\left[\mathrm{S}_{a u 1}, \mathrm{~S}_{a u 2}, \mathrm{~S}_{a u 3}\right]^{\mathrm{T}}$ is given by[17]:

$$
\boldsymbol{S}_{a u}= \begin{cases}l_{1} \boldsymbol{q}_{v}+l_{2} \operatorname{sig}\left(\boldsymbol{q}_{v}\right)^{2} & \text { if } \overline{\boldsymbol{S}} \neq 00_{3 \times 1},\left\|\boldsymbol{q}_{v}\right\| \leq \varepsilon \\ \alpha \operatorname{sig}\left(\boldsymbol{q}_{v}\right)^{g_{1}}+\beta \operatorname{sig}\left(\boldsymbol{q}_{v}\right)^{g_{2}} & \text { otherwise }\end{cases}
$$

where $\overline{\boldsymbol{S}}=\boldsymbol{w}+\alpha \operatorname{sig}\left(\boldsymbol{q}_{v}\right)^{g_{1}}+\beta \operatorname{sig}\left(\boldsymbol{q}_{v}\right)^{g_{2}} . \quad \alpha, \beta, g_{1}, g_{2}$ are positive constants, satisfying $0.5<g_{1}=f_{1} / f_{2}<1$, $g_{2}=f_{3} / f_{4}>1, \quad l_{1}=0.5 \alpha \varepsilon^{g_{1}-1}+0.5 \beta \varepsilon^{g_{2}-1}, l_{2}=0.5 \alpha \varepsilon^{g_{1}-2}+0.5 \beta \varepsilon^{g_{2}-2}, f_{1}<f_{2}, f_{3}>f_{4}$ are positive odd numbers. $\varepsilon$ is a small positive constant, for instance $\varepsilon=0.001$.

Remark 2: Because of $g_{1} \in(0.5,1), g_{2}>1$, if $\varepsilon=0.001, l_{2}$ is much bigger than $l_{1}$. Therefore, when $\left|\boldsymbol{q}_{v}\right| \leq \varepsilon, \quad l_{2} \operatorname{sig}\left(\boldsymbol{q}_{v}\right)^{2}$ has the same magnitude as $l_{1} \boldsymbol{q}_{v}$, so it is guaranteed that $l_{2} \operatorname{sig}\left(\boldsymbol{q}_{v}\right)^{2}$ takes effect to drive quaternion converging fast to sliding mode. Furthermore, the choice of $l_{1}$ and $l_{2}$ can ensure the function $\boldsymbol{S}_{a u}$ and its time derivative continuity. When $\left|\boldsymbol{q}_{v}\right|>\varepsilon, \quad \boldsymbol{S}_{a u}=\alpha \operatorname{sig}\left(\boldsymbol{q}_{v}\right)^{g_{1}}+\beta \operatorname{sig}\left(\boldsymbol{q}_{v}\right)^{g_{2}}$, it is guaranteed that sliding surface $S$ and $\bar{S}$ have the same form. Meanwhile, the fixed-time non-singular sliding mode surface reaches zero, $\boldsymbol{q}_{v}$ and $\boldsymbol{w}$ will approach zero along the surface (7).

Remark 3: The surface in [18] has 10 parameters need to design, the surface (7) only has 4 parameters need to design. Compare with the surface in [18], the sliding surface has fewer parameters, so, in actual use of (7) to design control law, the sliding surface (7) is more concise.

According to (7):

$$
\boldsymbol{J} \dot{\boldsymbol{S}}=\boldsymbol{J} \dot{\boldsymbol{w}}+\frac{\boldsymbol{J}}{2} \boldsymbol{F}\left(q_{0} \boldsymbol{I}_{3}+\boldsymbol{q}_{v}^{\times}\right) \boldsymbol{w}
$$

where

$$
\boldsymbol{F}= \begin{cases}l_{1} \boldsymbol{I}_{3}+2 l_{2} \operatorname{diag}\left(\operatorname{sign}\left(q_{v i}\right) q_{v i}\right) & \text { if } \overline{\boldsymbol{S}} \neq 0,\left\|\boldsymbol{q}_{v}\right\|<\varepsilon \\ \alpha g_{1} \operatorname{diag}\left(\operatorname{sign}\left(q_{v i}\right) q_{v i}^{g_{1}-1}\right)+\beta g_{2} \operatorname{diag}\left(\operatorname{sign}\left(q_{v i}\right) q_{v i}^{g_{2}-1}\right) & \text { otherwise }\end{cases}
$$

Remark 4: Because of $g_{1}=f_{1} / f_{2}$, so $g_{1}-1=\left(f_{1}-f_{2}\right) / f_{2}, f_{1}, f_{2}$ are positive odd number, so $f_{1}-f_{2}$ is an even number. So, we can get, when $q_{v i}<0, q_{v i}^{g_{1}-1}$ is a rational number. Similarly, $q_{v i}^{g_{2}-1}$ is also a rational number when $q_{v i}<0$. So $\mathrm{F}$ will not be singular for any $q_{v i}$. Substitutes (4) into (9), there is:

$$
\begin{gathered}
\boldsymbol{J} \dot{\boldsymbol{S}}=-\boldsymbol{w}^{\times} \boldsymbol{J} \boldsymbol{w}+\boldsymbol{D} \boldsymbol{u}_{c}(\mathrm{t})+\overline{\boldsymbol{d}}(t)+ \\
\frac{\boldsymbol{J}}{2} \boldsymbol{F}\left(q_{0} \boldsymbol{I}_{3}+\boldsymbol{q}_{v}^{\times}\right) \boldsymbol{w}
\end{gathered}
$$

The approximation law is designed as follows: 


$$
J \dot{S}=-K_{1} \operatorname{sig}^{\lambda}(S)-K_{2} \operatorname{sig}^{\gamma}(S)
$$

where $\boldsymbol{K}_{i}=\operatorname{diag}\left(K_{i 1}, K_{i 2}, K_{i 3}\right)(i=1,2) \quad$ is a diagonal matrix and $K_{i j}>0(j=1,2,3)$. For $\boldsymbol{x} \in \mathbf{R}^{3}$, $\operatorname{sig}^{\alpha}(\boldsymbol{x})=\left[\left|x_{1}\right|^{\alpha} \operatorname{sign}\left(x_{1}\right),\left|x_{2}\right|^{\alpha} \operatorname{sign}\left(x_{2}\right),\left|x_{3}\right|^{\alpha} \operatorname{sign}\left(x_{3}\right)\right]^{\mathrm{T}} \cdot \gamma=\gamma_{1} / \gamma_{2} \in(0,1), \lambda=\lambda_{1} / \lambda_{2}>1, \quad \gamma_{1}<\gamma_{2}, \quad \lambda_{1}>\lambda_{2} \quad$ are positive odd number.

Step 2: Designing the control law

Assumption 3: In order to design control law, we give the flowing assumption, where $c_{1} \geq 0$ and $c_{2} \geq 0$ :

$$
\begin{gathered}
\left\|-\boldsymbol{w}^{\times} \boldsymbol{J} \boldsymbol{w}\right\| \leq c_{1}\|\boldsymbol{w}\|^{2} \\
\left\|\frac{\boldsymbol{J}}{2} \boldsymbol{F}\left(q_{0} \boldsymbol{I}_{3}+\boldsymbol{q}_{v}^{\times}\right) \boldsymbol{w}\right\| \leq c_{2}\|\boldsymbol{w}\|
\end{gathered}
$$

Remark 5: Because of $\left\|q_{0} \boldsymbol{I}_{3}+\boldsymbol{q}_{v}^{\times}\right\|=1$ from Eq.(14), $\|F\| \leq c_{21}$ is satisfied, equations $\left\|\frac{\boldsymbol{J}}{2} \boldsymbol{F}\right\| \leq \frac{\lambda_{\eta}}{2} c_{21} \leq c_{22}$ and $\left\|\frac{\boldsymbol{J}}{2} \boldsymbol{F}\left(q_{0} \boldsymbol{I}_{3}+\boldsymbol{q}_{v}^{\times}\right) \boldsymbol{w}\right\| \leq\left\|\frac{\boldsymbol{J}}{2} \boldsymbol{F}\right\| \cdot\left\|\left(q_{0} \boldsymbol{I}_{3}+\boldsymbol{q}_{v}^{\times}\right)\right\|\|\boldsymbol{w}\| \leq c_{2}\|\boldsymbol{w}\|$ are also satisfied.

Remark 6: Through (13), if the inertia J of the spacecraft is disturbed, the influence caused by the uncertainties of that can be eliminated by self-adaptation of parameter $c_{1}$.

Lemma 1: Consider the nonlinear system [17]:

$$
\dot{x}(t)=f(x(\mathrm{t})), x(0)=0, f(0)=0, x \in \square^{n}
$$

Suppose that there is a Lyapunov function $V(\boldsymbol{x})$, and scalars $\alpha, \beta, p, q \in \square^{+}, \quad p<1, q>1$, such that

$$
\dot{V}(x)+\alpha V^{p}(x)+\beta V^{q}(x) \leq 0
$$

Then, the trajectory of this system is practical fixed-time stable, which means the convergence time is independent of the initial state, and the convergence time is given as:

$$
T \leq \frac{1}{\alpha(1-p)}+\frac{1}{\beta(q-1)}
$$

Theorem 1: According to the previous derivation, the control law designed as follows:

$$
\boldsymbol{u}_{c}(t)=\boldsymbol{D}^{\div}\left(-\boldsymbol{K}_{1} \operatorname{sig}^{\lambda}(\boldsymbol{S})-\boldsymbol{K}_{2} \operatorname{sig}^{\gamma}(\boldsymbol{S})-\frac{\boldsymbol{S}}{\|\boldsymbol{S}\|+\varepsilon}\left(\hat{c}_{1}\|\boldsymbol{w}\|^{2}+\hat{c}_{2}\|\boldsymbol{w}\|+\hat{b} \varphi\right)\right)
$$

where $\boldsymbol{D}^{\div}=\boldsymbol{D}^{\mathrm{T}}\left(\boldsymbol{D} \boldsymbol{D}^{\mathrm{T}}\right)^{-1}, \hat{c}_{1}, \hat{c}_{2}$ and $\hat{b}$ are adaptive parameters, $\varphi=1+\|\boldsymbol{w}\|+\|\boldsymbol{w}\|^{2}+\|\boldsymbol{w}\|^{2-\eta}+\|\boldsymbol{w}\|^{\eta}, \varepsilon$ is small positive constant. The $\hat{c}_{1}, \hat{c}_{2}, \hat{b}$ are selected as follows:

$$
\begin{gathered}
\dot{\hat{\hat{c}}}_{1}=\mu_{1}\|\boldsymbol{S}\|\|\boldsymbol{w}\|^{2} \\
\dot{\hat{\hat{c}}}_{2}=\mu_{2}\|\boldsymbol{S}\|\|\boldsymbol{w}\| \\
\dot{\hat{b}}=\mu_{3}\|\boldsymbol{S}\| \varphi
\end{gathered}
$$


where $\mu_{i}, i=1,2,3$ are design parameters.

Step 3: Proving the stability

Proof: In this section, select the following Lyapunov function:

$$
V_{1}=\frac{1}{2} \boldsymbol{S}^{\mathrm{T}} \boldsymbol{J} \boldsymbol{S}+\frac{1}{2 \mu_{1}} \tilde{c}_{1}^{2}+\frac{1}{2 \mu_{2}} \tilde{c}_{2}^{2}+\frac{1}{2 \mu_{3}} \tilde{b}^{2}
$$

where $\tilde{c}_{1}=c_{1}-\hat{c}_{1}, \quad \tilde{c}_{2}=c_{2}-\hat{c}_{2}, \quad \tilde{b}=b-\hat{b}$.

According to reference [18], the derivative of $V_{1}$ is as follows (For proof details, please refer to [18]):

$$
\begin{aligned}
\dot{V}_{1} \leq & \delta \boldsymbol{S}^{\mathrm{T}}\left(-\boldsymbol{K}_{1} \operatorname{sig}^{\lambda}(\boldsymbol{S})-\boldsymbol{K}_{2} \operatorname{sig}^{\gamma}(\boldsymbol{S})\right) \\
\leq & -\min \left(K_{1 i}\right)\left(\frac{2}{\lambda_{\max }(\boldsymbol{J})}\right)^{(\lambda+1) / 2}\left(\frac{1}{2} \boldsymbol{S}^{\mathrm{T}} \boldsymbol{J}\right)^{(\lambda+1) / 2}- \\
& \min \left(K_{2 i}\right)\left(\frac{2}{\lambda_{\max }(\boldsymbol{J})}\right)^{(\gamma+1) / 2}\left(\frac{1}{2} \boldsymbol{S}^{\mathrm{T}} \boldsymbol{J} \boldsymbol{S}\right)^{(\gamma+1) / 2} \leq 0 \\
\dot{V}_{1} \leq & -\left(\frac{2}{\lambda_{\max }(\boldsymbol{J})}\right)^{(\lambda+1) / 2}\left(\min \left(K_{1 i}\right)-\left(\frac{L_{1}}{V_{1}}\right)^{(\lambda+1) / 2}\right) V_{1}^{(\lambda+1) / 2}- \\
& \left(\frac{2}{\lambda_{\max }(\boldsymbol{J})}\right)^{(\gamma+1) / 2}\left(\min \left(K_{2 i}\right)-\left(\frac{L_{1}}{V_{1}}\right)^{(\gamma+1) / 2}\right) V_{1}^{(\gamma+1) / 2}
\end{aligned}
$$

where $L_{1}=\frac{1}{2 \mu_{1}} \tilde{c}_{1}^{2}+\frac{1}{2 \mu_{2}} \tilde{c}_{2}^{2}+\frac{1}{2 \mu_{3}} \tilde{b}^{2}$. And $\frac{L_{1}}{V_{1}}<1,\left(\frac{L_{1}}{V_{1}}\right)^{(\lambda+1) / 2}<1,\left(\frac{L_{1}}{V_{1}}\right)^{(\gamma+1) / 2}<1$ according to $L_{1}<V_{1}$. If $\min \left(K_{1 i}\right) \geq 1, \quad \min \left(K_{2 i}\right) \geq 1$. Expression (24) is simplified into $\dot{V}(x)+\alpha V^{p}(x)+\beta V^{q}(x)$; So we can get:

$$
t_{1} \leq \frac{1}{\chi_{2}(1-p)}+\frac{1}{\chi_{1}(q-1)}
$$

where $\quad \chi_{1}=\delta\left(\frac{2}{\lambda_{\max }(\boldsymbol{J})}\right)^{(\lambda+1) / 2}\left(\min \left(K_{1 i}\right)-\left(\frac{L_{1}}{V_{1}}\right)^{(\lambda+1) / 2}\right) \quad$ and $\quad \chi_{2}=\delta\left(\frac{2}{\lambda_{\max }(\boldsymbol{J})}\right)^{(\gamma+1) / 2}\left(\min \left(K_{2 i}\right)-\left(\frac{L_{1}}{V_{1}}\right)^{(\gamma+1) / 2}\right)$, $p=\frac{\gamma+1}{2}<1, q=\frac{\lambda+1}{2}>1$.

Theorem 2: When the spacecraft attitude reach the sliding mode $S=0$, the attitude errors will converge to zero in a fixed-time.

Proof:. Select the following Lyapunov function:

$$
V_{2}=0.5\left[\boldsymbol{q}_{v}^{T} \boldsymbol{q}_{v}+\left(1-q_{0}\right)^{2}\right]
$$

Based on $\boldsymbol{q}_{v}^{\mathrm{T}} \boldsymbol{q}_{v}^{\times}=0$, then

$$
\begin{aligned}
\dot{V}_{2} & =\boldsymbol{q}_{v}^{\mathrm{T}} \dot{\boldsymbol{q}}_{v}-\left(1-q_{0}\right) \dot{q}_{0} \\
& =0.5 \boldsymbol{q}_{v}^{\mathrm{T}}\left(q_{0} \boldsymbol{I}_{3}+\boldsymbol{q}_{v}^{\times}\right) \boldsymbol{w}+0.5 \boldsymbol{q}_{v}^{\mathrm{T}}\left(1-q_{0}\right) \boldsymbol{w} \\
& =0.5 q_{0} \boldsymbol{q}_{v}^{\mathrm{T}} \boldsymbol{w}+0.5 \boldsymbol{q}_{v}^{\mathrm{T}} \boldsymbol{w}\left(1-q_{0}\right)+0.5 \boldsymbol{q}_{v}^{\mathrm{T}} \boldsymbol{q}_{v}^{\times} \boldsymbol{w} \\
& =0.5 \boldsymbol{q}_{v}^{\mathrm{T}} \boldsymbol{w} \\
& =-0.5 \alpha \boldsymbol{q}_{v}^{\mathrm{T}} \operatorname{sig}\left(\boldsymbol{q}_{v}\right)^{g_{1}}-0.5 \beta \boldsymbol{q}_{v}^{\mathrm{T}} \operatorname{sig}\left(\boldsymbol{q}_{v}\right)^{g_{2}}
\end{aligned}
$$


So, we obtain $\lim _{t \rightarrow t_{2}} q_{0}=1$ because $\left(1-q_{0}\right)^{2} \leq\left(1-q_{0}\right)\left(1+q_{0}\right)=\boldsymbol{q}_{v}^{T} \boldsymbol{q}_{v}$, therefore:

$$
V_{2} \leq \boldsymbol{q}_{v}^{T} \boldsymbol{q}_{v}
$$

According to (27), formula (26) can be transformed into:

$$
\dot{V}_{2}+\chi_{1} V_{2}^{\left(g_{1}+1\right) / 2}+\chi_{2} V_{2}^{\left(g_{2}+1\right) / 2} \leq 0
$$

where $\chi_{1}=0.5 \alpha, \chi_{2}=0.5 \beta$. Let $t_{2}$ be the reaching time, then $t_{2} \leq \frac{2}{\chi_{1}\left(1-g_{1}\right)}+\frac{2}{\chi_{2}\left(g_{2}-1\right)}$.

\section{Simulation Results Analysis}

In this paper, the parameters of spacecraft are selected as follows (Table 1):

Table 1. Spacecraft Parameters

\begin{tabular}{ll}
\hline \hline parameter & value \\
\hline Nominal moment of inertial & $\boldsymbol{J}=\operatorname{diag}[6 ; 7 ; 9] \mathrm{kg} \cdot \mathrm{m}^{2}$ \\
$\boldsymbol{Q}(0)$ & $\boldsymbol{Q}(0)=[0.854,0.475,0.095,0.19,]^{\mathrm{T}}$ \\
$\boldsymbol{w}(0) /\left(\mathrm{rad} \cdot \mathrm{s}^{-1}\right)$ & $\boldsymbol{w}(0)=[-0.25,0.5,-0.15]^{\mathrm{T}} \mathrm{rad} / \mathrm{s}$ \\
$d(t)$ & $\boldsymbol{d}(t)=\left(\boldsymbol{w}^{2}+0.005\right)[0.1 \sin (0.1 t), 0.2 \sin (0.2 t), 0.3 \sin (0.3 t)]^{\mathrm{T}} \mathrm{N} \cdot \mathrm{m}$ \\
\hline
\end{tabular}

The actuator effective decline faults are:

$$
E_{i}= \begin{cases}1 & t \leq 5 \\ 0.5+0.1 \sin (0.5 t+\pi i / 3) & t>5\end{cases}
$$

The actuator drift faults are:

$$
\bar{u}_{c i}= \begin{cases}0 & t \leq 15 \\ 0.1+0.05 \sin (0.5 i \pi t) & t>15\end{cases}
$$

The control torque distribution matrix is:

$$
D=\left[\begin{array}{llll}
1 & 0 & 0 & 1 / \sqrt{3} \\
0 & 1 & 0 & 1 / \sqrt{3} \\
0 & 0 & 1 & 1 / \sqrt{3}
\end{array}\right]
$$

The parameters of the FNTSM control law (18) and the sliding mode (7) are selected as follows: $\boldsymbol{K}_{1}=0.5 \operatorname{diag}[1,1,1], \quad \boldsymbol{K}_{1}=0.3 \operatorname{diag}[1,1,1], \quad \gamma=5 / 7, \lambda=9 / 5 \quad \eta=1.9, \quad \mu_{1}, \mu_{2}=0.8, \quad \mu_{3}=0.4, \quad c_{10}=0.01$, $c_{20}=0.01, b_{0}=0.01, \alpha=0.54, \beta=0.73, g_{1}=25 / 27, g_{2}=19 / 5, \varepsilon=0.01$. The max controlling torque is $u_{\max }=2.0 \mathrm{Nm}$.

Fig. 1 depicts the attitude quaternion. From Fig. 1, we can see that the spacecraft attitude cans quickly stability in a short time. As can be seen from Fig. 1, the spacecraft attitude from the initial state to the process of achieving attitude stability, the change of spacecraft attitude is very stable without attitude fluctuations. 
Fig. 2 gives the response curves of angular velocity. From Fig. 2, it can be seen that in the course of attitude stabilization, the attitude angular velocity curve changes steadily, and the attitude angular velocity stabilization can be achieved quickly.

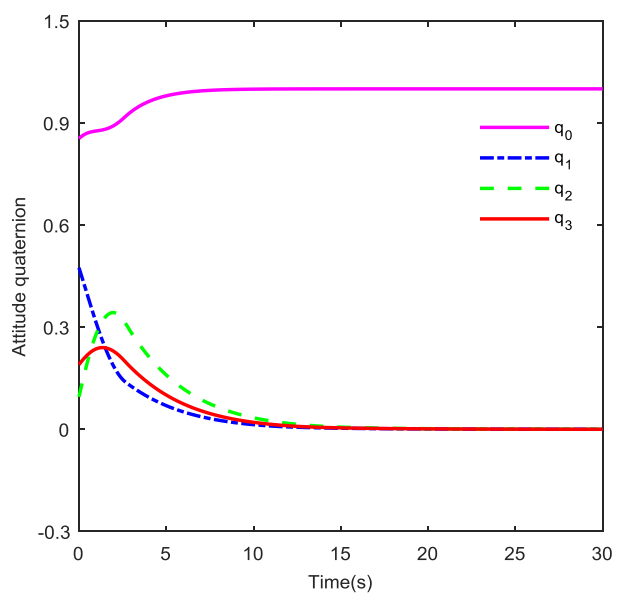

Fig. 1. Time response of attitude quaternion.

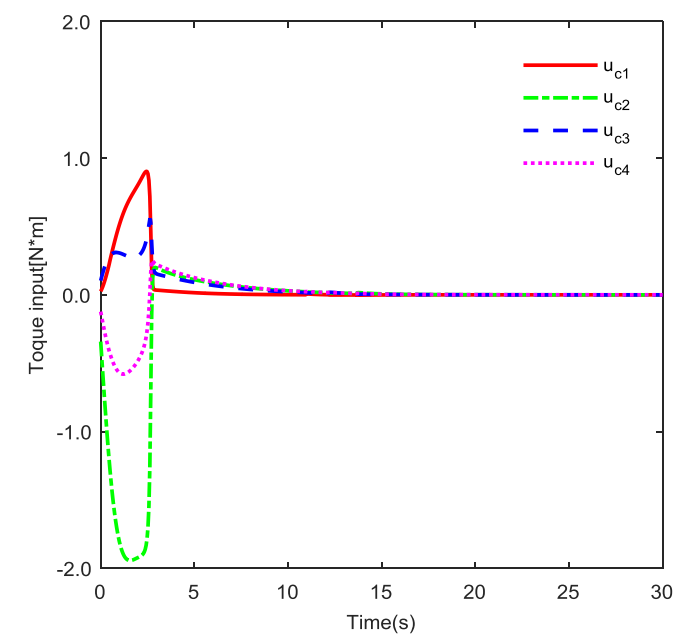

Fig. 3. Time response of controlling torque.

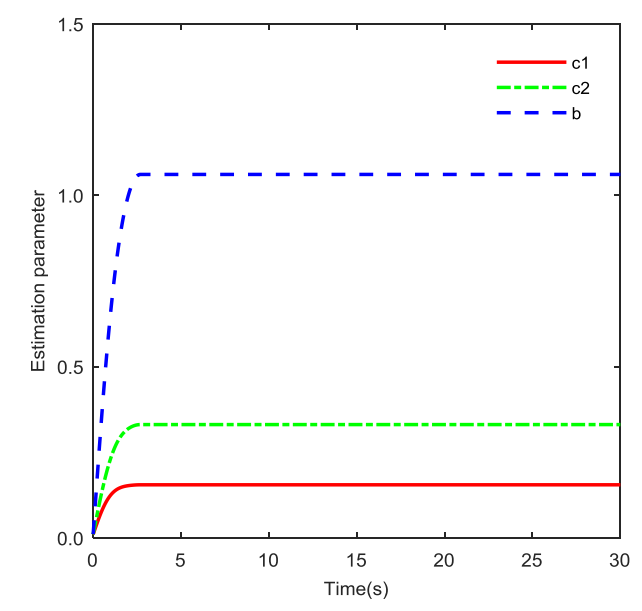

Fig. 5. Time response of estimating parameters.

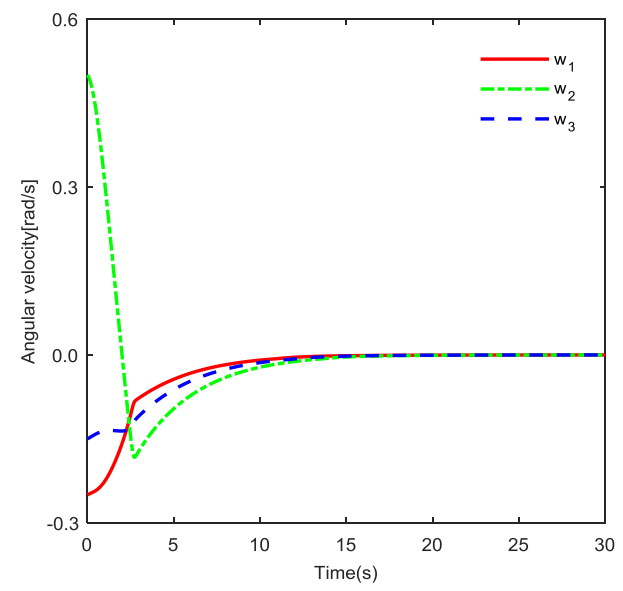

Fig. 2. Time response of angular velocity.

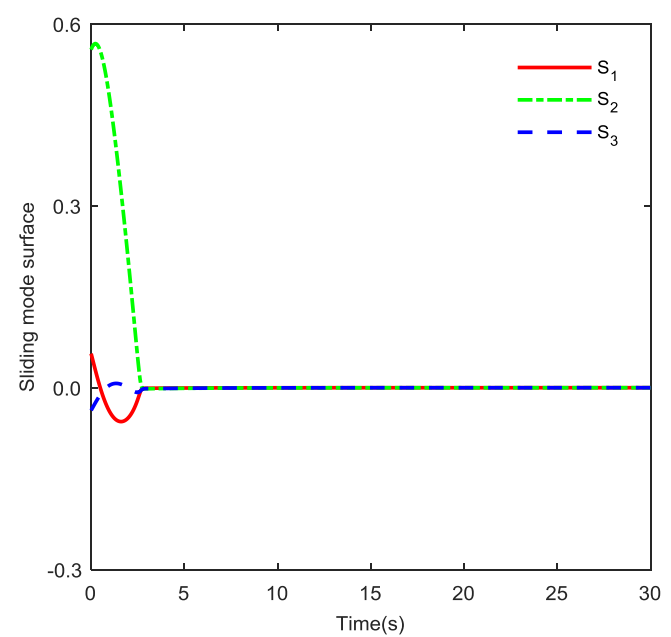

Fig. 4. Time response of estimating unknown parameters and controller saturation.

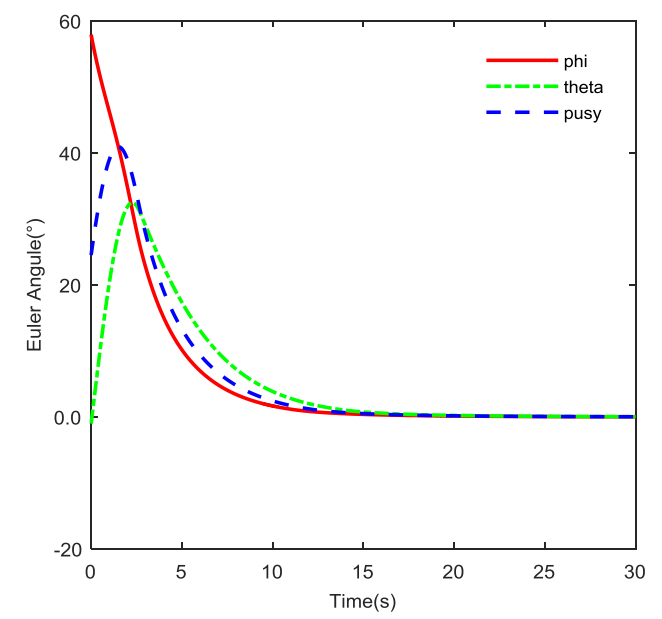

Fig. 6. Time response of Euler angles. 
Fig. 3 shows the control torque curve. It can be seen from the figure that the system does not saturate during the entire control process. In addition, the fluctuation frequency is relatively low and the curve changes very smoothly. Therefore, the control law designed in this paper has superior control performance.

Fig. 4 gives the response curves of sliding mode surface. From Fig. 4, we can see that the FNTSM control law can quickly reach the sliding mode surface.

Fig. 5 gives the estimated curves of uncertainty parameters. It is shows that the adaptive parameters designed in this paper can achieve stability in a relatively short time.

Fig. 6 gives the change curves of Euler angles. From Fig. 6, we can see that the attitude angle of the spacecraft can be stabilized from the initial state.

\section{Conclusion}

Fixed-time convergence control strategies based on adaptive Non-Singular Terminal Sliding Mode are proposed for rigid spacecraft attitude stabilization subject to actuator faults. Consider the actuator faults affecting the spacecraft, the adaptive FNTSM law we proposed can guarantee both the error quaternion and the angular velocity error convergence in finite time.

\section{Acknowledgment}

Financial support of this work by QIAN Xuesen Young Innovation Foundation, CASC. The authors would like to thank Hui Fu, Fanghao Tian and Hang Qian for their comments and suggestions that helped to improve the paper significantly.

\section{References}

[1] $\mathrm{Hu}, \mathrm{Q} ., \mathrm{Li}, \mathrm{B} .$, \& Zhang, A. (2013). Robust finite-time control allocation in spacecraft attitude stabilization under actuator misalignment. Nonlinear Dynamics, 73(1-2), 53-71.

[2] Pukdeboon, C., Zinober, A. S. I., \& Thein, M. L. (2010). Quasi-continuous higher order sliding-mode controllers for spacecraft-attitude-tracking maneuvers. IEEE Transactions on Industrial Electronics, 57(4), 1436-1444.

[3] Yeh, F. (2010). Sliding-mode adaptive attitude controller design for spacecraft with thrusters. IET Control Theory and Applications, 4(7), 1254-1264.

[4] Jin, J., Ko, S., \& Ryoo, C. (2008). Fault tolerant control for satellites with four reaction wheels. Control Engineering Practice, 16(10), 1250-1258.

[5] Xiao, B., Huo, M., Yang X., \& Zhang, Y. (2015). Fault tolerant attitude stabilization for satellite without rate sensors. IEEE Transactions on Industrial Electronics, 62(11), 7191-7202.

[6] Yu, S., \& Long, X. (2015). Finite-time consensus for second-order multi-agent systems with disturbances by integral sliding mode. Automatica, 54, 158-165.

[7] Wang, X., \& Wang, J. (2015). Partial integrated guidance and control with impact angle constraints. Journal of Guidance, Control, and Dynamics, 38(5), 925-936.

[8] Li, P., Ma, J., \& Zheng, Z. (2016). Robust adaptive multivariable higher-order sliding mode flight control for air-breathing hypersonic vehicle with actuator failures. International Journal of Advanced Robotic Systems, 13(5), 1-12.

[9] Li, P., Ma, J., Geng L., \& Zheng, Z. (2015). Robust and adaptive non-homogeneous higher order sliding mode control for a class of uncertain nonlinear systems. Proceedings of the 34th Chinese Control Conference (pp. 3327-3334). HangZhou, China.

[10] Tiwari, P. M., Janardhanan, S., \& Un-Nabi, M. (2015). Rigid spacecraft fault-tolerant control using adaptive fast terminal sliding mode. In A. T. Azar, \& Q. Zhu (Eds.), Advances and Applications in Sliding 
Mode Control Systems (pp. 381-406). Switzerland: Springer.

[11] Lu, K. (2014). Research on Compound Control Design Methods for Spacecraft Attitude. PhD dissertation, Beijing Institute of Technology, Beijing.

[12] Lu, K., Xia, Y., \& Fu, M. (2013). Controller design for rigid spacecraft attitude tracking with actuator saturation. Information Sciences, 220, 343-366.

[13] Lu, K., \& Xia, Y. (2012). Finite-time fault-tolerant control for rigid spacecraft with actuator saturations. IET Control Theory and Applications, 7(11), 1529-1539.

[14] Gui, H., \& Vukovich, G. (2015). Adaptive integral sliding mode control for spacecraft attitude tracking with actuator uncertainty. Journal of the Franklin Institute, 352(12), 5832-5852.

[15] Wang, H., Hu, Q., Shi, Z., \& Gao, J. (2015). Back stepping-based finite-time fault-tolerant attitude tracking control for spacecraft. Acta Aeronautica et Astronautica Sinica, 36(6), 1933-1939.

[16] Xiao, B., Hu, Q., \& Zhang, Y. (2014). Finite-time attitude tracking of spacecraft with fault-tolerant attitude capability. IEEE Transactions on Control Systems Technology, 23(4), 1338-1350.

[17] Jiang, B., Hu, Q., Friswell, \& M. I. (2016). Fixed-time attitude control for rigid spacecraft with actuator saturation and faults. IEEE Transactions on Control Systems Technology, 24(5), 1892-1898.

[18] Han, Z., Zhang, K., Yang, T., \& Zhang, M. (2016). Spacecraft fault-tolerant control using adaptive non-singular fast terminal sliding mode. IET Control Theory and Applications, 10(16), 1991-1999.

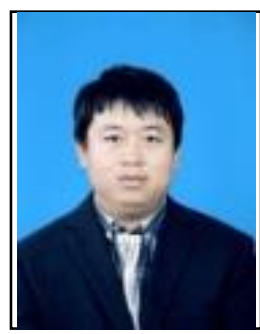

Zhiguo Han, who was born in Xi'an, Shaanxi province, on 28th March 1986, has fulfilled both his master's and doctor's degree in 2013 and 2017 respectively in Northwestern Polytechnical University (NWPU). He is currently a teacher in NWPU. His research interest contains control engineering, fault-tolerant control and deep learning.

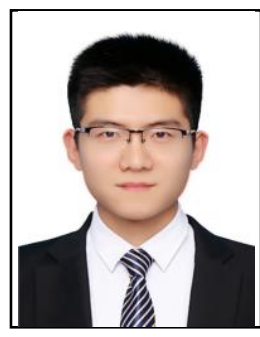

Hui Fu, who was born in Xi'an, Shaanxi province, 1991, has fullfilled both his bachelor's and master's degree in 2014 and 2017 respectively in Northwestern Polytechnical University (NWPU). He is currently a Phd student in NWPU. His research interest contains control engineering, deep learning and signal processing.

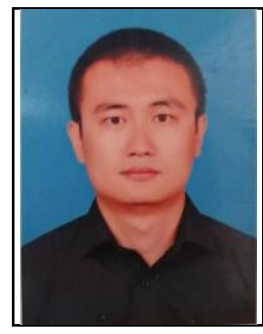

Fanghao Tian, who was born in Baoji, Shaanxi province, on 1st January 1995, has fulfilled his bachelor's degree in Northwestern Polytechnical University (NWPU) in 2017. He is currently a master student in NWPU. His research interest contains control engineering, deep learning and signal processing. 